\title{
The effect of HIV/AIDS on household's healthcare expenditure and income in Addis Ababa: a propensity score matching approach
}

\author{
Solomon Kibret \\ University of Insubria, Varese, Italy
}

\begin{abstract}
Introduction: The focus of this paper is to assess the effect of HIV/AIDS on household healthcare expenditure and income by comparing HIV-affected households with non-affected ones.

Material and methods: Primary data was collected using structured and pretested questionnaires in Addis Ababa, Ethiopia, in the period between January and February 2015. A total of 240 households were interviewed, 149 of which were HIV-affected households and the remaining 91 were non-affected. Since the sample of HIV-affected households was non-random and there is a strong risk of selection biases because of pre-existing differences between the two groups, direct comparisons of the outcomes may be misleading. This is because the existence of confounding factors creates biases in the estimation of average treatment effects on the outcome variables. To reduce this bias and to control confounding factors, propensity score matching methods were employed.

Results: The total monthly health expenditure of the affected households were on average 375 Ethiopian Birr (about \$ 18) higher than the non-affected households. The share of health expenditure on total expenditure was also found approximately 14 percentage point higher than the non-affected. On the other hand, the monthly per capita income, the share of expenditure on food were considerably lower among the affected households. The average treatment effect on the treated for the share of expenditure on food was 13 to 19 percentage point lower. The HIV-affected households had lost, on average, 6 more workdays than the non-affected.

Conclussion: The study concluded that the economic burden of HIV falls mainly on the affected household members and their families. Some policy measures should be designed targeting the affected households to mitigate the economic burden arises from HIV/AIDS and to improve the household's welfare. Alternative social support mechanisms like healthcare financing in the form of prepayment and strengthening households' economic activities by creating job opportunities and facilitating loan services from micro credit associations would mitigate the burden for households.
\end{abstract}

HIV AIDS Rev 2018; 17, 2: 103-110 DOI: https://doi.org/10.5114/hivar.2018.76369

Key words: HIV/AIDS, burden, matching, expenditure, income, Addis Ababa, illness, households.

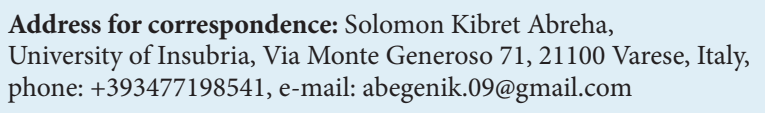

Address for correspondence: Solomon Kibret Abreha, University of Insubria, Via Monte Generoso 71, 21100 Varese, Italy, phone: +393477198541, e-mail: abegenik.09@gmail.com

Article history:

Received: 09.04.2017

Received in revised form: 13.01.2018

Accepted: 16.01.2018

Available online: 21.05.2018 


\section{Introduction}

Many studies indicated ill health as the main contributor to many household impoverishments especially in developing countries [1-4]. The emergence of HIV/AIDS and other endemic diseases aggravating the problem of the health system in general and the poor households in particular. The increasing health care expenditure especially in developing countries without effective health insurance policies, cause a substantial challenge to the economic sustainability of the households [3].

Despite the severity of the problem in developing countries like Ethiopia, systematic studies on the economic burden of illness in general and HIV/AIDS epidemic at the micro level are very rare. Most studies in the developing countries focused on the macro level economic impact of diseases. However, other studies suggested that besides assessments of the macro level impact of illness, there should be a research interest on the micro impact by focusing on the household [3]. Russell [3] also noted further micro economic research on the household burden of illness studies and their implication to the economic development and effectiveness of the health system performance.

Evidence suggested that the interest of the cost of illness of the household has emerged mainly from the household production of health. Different cost of illness studies [2, 5] also concluded that the household was the appropriate unit of analysis to study the economic burden of illness. The reason for this was most of the economic burden of illness were borne by healthy household members.

HIV/AIDS epidemic is one of the major killer epidemics and affected many peoples in sub-Saharan countries. Evidence showed there are several ways in which HIV/ AIDS affects the household negatively. One is HIV/AIDS reduced non-health consumption expenditures (food expenditures) among household members [6]. Food consumption in an AIDS-affected household could decrease by $40 \%$, and this leads the children to higher risk of malnutrition [7]. Household members bear most of the burden because they are the primary units for coping with the disease and its consequences. The HIV-affected household member is the breadwinner that suffer financially, both from the loss of earnings and from the increased expenditure for medical care. The loss of income and the opportunity cost of caring for a family member impoverish households in the long run situations. Studies document reduced levels of household consumption, including a reduction in food consumption, resulting in malnutrition.

Evidence also showed that there were significant losses of household income due to terminal HIV/AIDS illness. A study in Eastern Zimbabwe [7] supported the evidence, in which the household member was lead to significant losses of income. The additional pressure exerted on the household coupled with the inadequate incomes and increased healthcare costs. The study indicated that because of these financial pressures, many children in the HIV-affected household member are forced to drop out of school. Among them, the majority are girls. These girls stay at home to do homework or care for their families, and they face an increased risk of engaging in hazardous labor and of being otherwise exploited. To do so, they scarify their education and participation in their community and other social life activities [7].

Reallocation of household efforts away from income earning activity to care-giving roles is another effect of HIV/AIDS to the affected households [6]. This is because HIV/AIDS has the possibility of incurring large expenditure [6] on treating affected household members and time spent for care-giving with HIV and other expenses like funeral expenses. The lack of adequate coping strategies aggravates the problem $[6,8]$. These effects are exacerbated when drugs for the treatment of HIV/AIDS are expensive, and public subsidies for care provision remain limited, or if health insurance is unavailable to affected households $[6,8]$.

Children life is affected in many ways among the HIVaffected household members. Evidenced by different studies indicated that many children become orphaned as they lose one or both of their parents due to HIV. According to UNICEF, 15 million children under the age of 18 had been orphaned by HIV/AIDS in 2003 [7]. AiDS Orphan 2013 report [8] estimated that worldwide AIDS has orphaned 17.8 million children under 18 years of age. According to the report in 2011, about 230,000 children under the age of 15 died because of HIV-related illnesses [8]. The study showed that around 15.1 million or $85 \%$ of these children live in sub-Saharan Africa. In some countries, which are severely affected by the epidemic, a significant percentage of all orphaned children (for instance, $74 \%$ in Zimbabwe and 63\% in South Africa) are orphaned due to AIDS [8].

In general, the above evidence indicated that in many ways, the effect of HIV/AIDS would incur additional burden for the affected household members. Among some of the problems mentioned above like increasing health expenditure, the reduction of non-health expenditures, the loss of income to the household member, and the increasing number of AIDS orphan children will aggravate problems of health system mostly in developing countries. Poor households in these countries will suffer from the double burden of diseases and lead them to further impoverishment and poverty. The inequalities between the poor and the rich, and the health and the severely ill people will increase through time. In order to solve these problems, there should be an effective policy options by the health system and the government. This includes improving the health of the population and proving financial protection against the costs of ill health as well as equal access to healthcare to all population. This paper addressed problems mentioned above by investigating the HIV/AIDS-affected households at microeconomic level and suggesting alternative policy options to improve the health of these households. 


\section{HIV/AIDS in Ethiopia}

HIV was first identified in Ethiopia in 1984, and the first two AIDS cases were reported in 1986 [9]. Since then, the disease has spread at an alarming rate in all parts of the country. The latest EPP/spectrum modelling in 2013 reported in Ethiopia that there were an estimated 793,700 people living with HIV, including 200,300 children [9]. The spectrum indicated that there were approximately 45,200 AIDS-related deaths in 2013. HIV adult prevalence rate was estimated at $1.5 \%$ in 2011 [10]. According to the 2011 EDHS, adult prevalence was almost twice as high among females compared to males at $1.9 \%$ versus $1.0 \%$, respectively.

HIV transmission in Ethiopia is mainly through heterosexual contacts. Other ways of transmission also occur from mother to child, and through transfusion of infected blood and unsafe medical practices. The 'AIDS in Ethiopia' 2005 report estimated that $45 \%$ of Ethiopia's population under 15 years of age are especially vulnerable to the epidemic [9]. Further vulnerable population groups include female sex workers, long-distance truck drivers, migrant workers, unemployed people, and internally displaced populations [9]. The HIV epidemic in Ethiopia is becoming more concentrated in urban areas and along major transport corridors [9]. In Ethiopia, the spread of HIV/AIDS started and was first confined in major urban areas located along major roads and commercial routes. The Ethiopia DHS 2011 data shows HIV prevalence in large towns including Addis Ababa, the regional capital increased from 2005 to 2011 [11]. Higher prevalence in Addis Ababa and large towns may be associated with labor migration to large urban areas and large-scale construction projects as well as a growing service industry [9].

HIV/AIDS is one of the key challenges for overall national development and the health system in Ethiopia. WHO on its report of AIDS in Ethiopia in 2005 reported that AIDS led to a seven-year loss in life expectancy, close to a million orphans, and a loss of productivity and income at the workplace with severe effects at the household and community levels [9]. The high rates of mortality and morbidity associated with HIV/AIDS have strongly affected the health sector and are among the major impediments to delivering quality care to its full capacity [9].

In Ethiopia, a study of 25 AIDS-afflicted rural families found that the average health, funeral, and mourning expenses amounted to several times the average household income. Net farm income varies from 270 to 620 birr, depending on the region [12]. Selling productive assets, especially livestock, usually pays for these expenses. A recent study by the World Bank indicated that the average cost in sub-Saharan Africa to provide basic care to reduce suffering and to treat the less expensive opportunistic infections is about the U.S. $\$ 300$ per patient year. If all opportunistic infections are treated, the annual cost rises to U.S. \$500 [10]. Reports also show that HIV-affected households earned less income due to the illness. A study indicated that there was a difference in average annual income amounted 1,000 birrs between HIV-affected and non-affected households [12].
A data collected on absenteeism and medical cost found that $53 \%$ of all illness accounted for HIV/AIDS-related illness from the total 13,363 incidents over a five-year period [12]. Out of 19 individuals interviewed in detail, 11 lost 30 days over one year due to HIV/AIDS illness, 7 lost on average 60 days, while one person said he was absent for 240 days because of HIV/AIDS illness [12]. These figures indicated that days of absence because of HIV/AIDS illness are significantly high in Ethiopia.

The 2005 AIDS in Ethiopia report indicated that there is an increase in the number of orphans because of AIDS deaths to men and women. According to the report, the number of orphans due to AIDS is also growing and worsening the social and economic situation of children. As a result, in 2005, 4,885,337 orphans aged between 0-17 years were estimated [9]. Of these, 744,100 were AIDS orphans. From the total number of AIDS orphans, 529,777 were maternal, 464,506 paternal, and 250,195 dual orphans ${ }^{1}$ [8]. The $6^{\text {th }}$ AIDS report in 2005 reported that the total number of AIDS orphans in Ethiopia increased until 2010, although the rate of the increase is expected to lessen due to the impact of planned ART services. In 2013, the number of AIDS orphan was 898,000 [9]. There will be a remarkable strain on social systems to cope with such a large number of orphans and provide them with appropriate care and supervision.

At the family level, there will be an increased burden and stress for extended family. This surge in the number of orphans is especially difficult in a major urban center, where traditional family structures are not as strong as in the countryside [10]. A study in Addis Ababa showed that the burden of their care falls on grandparents, older sibling, and the community at large. The magnitude of the problem and the general level of poverty have weakened social cohesion and traditional coping mechanisms. Many orphans will never receive adequate health care and schooling, increasing the burden on society in future years. The number of street children will rise, and child labor will become more common as orphans look for ways to survive. Ethiopian strategic plan for intensifying multi-sectorial HIV/AIDS response report in 2004 reported that providing care and support to orphans has overwhelmed traditional coping capacity, leaving many children without their basic social educational needs and rights unattended, which in turn worsened their vulnerability.

There is an evidence on costs related to HIV/AIDS in Ethiopia. The study in Addis Ababa showed that HIV/ AIDS treatment and related infections resulting from AIDS is expensive and places substantial strains on the delivery of health services in Ethiopia. For example, the demand for health services because of AIDS results in hospital bed occupancy [10]. For those persons living with AIDS who need hospital care, the average length of stay in a hospital is longer than for most other diseases. The growing number of AIDS cases, people living with HIV/AIDS, orphans, oth-

${ }^{1} \mathrm{~A}$ maternal orphan is a child under age 15 whose mother has died from AIDS; a paternal orphan is a child under 15 whose father has died from the disease; a dual orphan is a child under 15 whose parents have both died from AIDS. 
er vulnerable children, and their continued needs for health care services have placed a significant burden on resources in the already inadequate health services. General primary health service coverage is already insufficient, and lack of health professionals to meet needs of community-based and another insurance mechanism for these needy patients has contributed significantly to the lack of an appropriate care and support response.

In short, HIV/AIDS has now become a serious threat and challenge to Ethiopia's socioeconomic development in general and Addis Ababa in particular. The epidemic is affecting the social and economic growth of the country by increasing poverty and by affecting the healthcare system. This paper mainly focuses on the economic costs and consequences of HIV/AIDS illness for the household in the study area of Addis Ababa, Ethiopia as well as to identify the main economic burden of HIV/AIDS on the patient members of the household by comparing HIV-affected households with the non-affected one.

\section{Methods}

This study exploits propensity score matching (PSM), a technique first proposed by Rosenbaum and Rubin [13]. They defined the propensity score as "the conditional probability of assignment to a particular treatment given a vector of observed covariates". As noted by Rosenbaum and Rubin [13] in randomized experiments, the results in the two treatment groups may often be directly compared because their units are likely to be similar. Whereas in non-randomized experiments, such direct comparisons may be misleading because the units exposed to one treatment generally differ systematically from the units exposed to the other treatment [13].

Average treatment effect (ATE): The $N$ units in the study are viewed as a simple random sample from some population, and the quantity estimated is the average treatment effect, defined the difference between the outcomes of treated and control observations [13].

$$
\begin{gathered}
\Delta=Y_{1}-Y_{2} \\
A T E=E(\Delta)=E\left(Y_{1} \mid X, D=1\right)-E\left(Y_{0} \mid X, D=0\right)
\end{gathered}
$$

where $E$ (.) denotes expectation in the population.

ATE is fine for random experiments but in observational studies, it may be biased if treated and control observations are not similar. Balancing scores can be used to group treated and control units, so that direct comparisons are more meaningful. A balancing score, $b(\mathrm{x})$, is a function of the observed covariates for a particular household $x$ such that the conditional distribution of $\mathrm{x}$ given $b(\mathrm{x})$ is the same for treated $(z=1)$ and control $(z=0)$ units; that is, in David's notation [13]:

$$
\mathrm{x} \perp \mathrm{z} \mid b(x)
$$

The simplest balancing score is certainly $b(x)=x b(x)=x$ which means that units are balanced only if they have the same values for the covariates. The algorithm of PSM can be stated as follows: suppose $N$ cases with an observed outcome $Y_{i}$ and a covariate vector $X_{i}=\left\{\mathrm{X}_{i 1}, \ldots \ldots . \mathrm{X}_{i} \mathrm{~K}\right\}$, where $_{i}=1, \ldots N$ and $K$ is the number of covariates. Let $Z_{i}$ be the treatment condition, with $Z_{i}=1$ a treatment group, and $Z_{i}=0$ for a control group. Rosenbaum and Rubin [13] defined a propensity score for the ${ }_{i}{ }_{i}^{\text {th }}$ case as follows:

$$
p\left(X_{i}\right)=\operatorname{pr}\left(Z_{i}=1 \mid X_{i}\right)
$$

The function $p\left(X_{i}\right) p\left(X_{i}\right)$ is called "the propensity score", that is, the propensity towards exposure to treatment 1 given the observed covariates. Propensity scores are the predicted probabilities obtained by using a logit or probit regression relative to the set of covariates.

The central piece of PSM is matching. Bai [14] defined matching as "a procedure to pair treatment and comparison groups with similar observable characteristics in order to reduce the estimation bias from the influence of unbalanced covariates". There are a variety of matching methods but the most commonly used matching methods are the following:

Nearest neighbor matching: matches each treated case (e.g. HIV patients) with a non-treated case (e.g. non-HIV individuals) with the closest absolute distance of their propensity scores [14]. In this case, it matches each case $i$ in the HIV patient group with a case $j$ in the non-HIV individuals with the closest absolute distance.

$$
d(i, j)=\mid l\left(X_{i}\right)-l\left(X_{j}\right)
$$

Caliper (radius) matching: matches each treated case within a pre-specified band, called caliper [14]. For each case $i$ in the HIV patient group with a case $j$ in the control group within a pre-specified band $b$. Cochran and Rubin recommend $b=0.25$ standard deviations of the logit of the propensity scores [14].

Mahalanobis metric matching: matches each treated case with a non-treated case with the closest Mahalanobis distance calculated based on proximities of the variables. It matches each case $i$ in the HIV patient group with a case $j$ in the non-HIV group with the closest Mahalanobis distance:

$$
D(i, j)=\left(Z_{i}^{T}-Z_{j}^{T}\right)^{T} \times S^{-1} \times\left(Z_{i}^{T}-Z_{j}^{T}\right)
$$

where $Z .(.=i$ or $j)$ is a new vector $(\mathrm{X} . l(\mathrm{X})$.$) , and S$ is the sample variance-covariance matrix of the new vector for the control group [14].

Stratification matching: classifies all the cases into several strata based on the corresponding number of percentiles of the propensity scores.

The main assumption of this study is conditional on the propensity score, where assignment to the treatment and control groups can be taken to be random [6]. In this case, the difference in outcomes between treatment and control groups can be directly compared to give the effect of HIV/ AIDS on the households. 
Let $X$ denotes the vector of observed covariates for a particular household and binary variable $z(1 / 0)$ indicates whether the household was HIV patient or not. Then, the propensity score $e(X)$ can be written $[13,15]$ as:

$$
e(x)=\operatorname{pr}(z=1 / \mathrm{x})=E(Z \mid X)
$$

The above equation is considered as the conditional probability of HIV patient given the covariates X. HIV patient households and non-HIV households selected to have the same value of $e(X)$ will have the same distribution of $X$ $[6,15] . Z$ and $X$ are conditionally independent given $e(X)$. Matching $e(X)$ tends to balance the distribution of $X$ in the matched sample between HIV patient and non-HIV patient groups.

\section{Variables in the study}

Treatment variable: the treatment variable used for this study is the health status of the household as 1 if HIV patient (treatment group) and 0 otherwise (control group). Propensity score matching will help to balance propensity scores of the treatment and control groups, so that direct comparisons of the data will be more meaningful once the groups are balanced on the covariates.

Explanatory variables: the variables used in this study were measured at the household level, since the study is focused on the impact of HIV/AIDS at the household level. The list of explanatory variables used for matching includes household head's characteristics such as age, education [6], occupation, gender, and marital status. Since variables like household income, household family size, and health expenditure are likely to be influenced by HIV status, they are not employed as covariates [6]. Therefore, the list of explanatory variables to match the propensity score are the gender ( 1 if male, 0 otherwise), age in years, education level, and occupation dummy (no occupation, employed by government, NGO employee, self-employed).

Outcome variables: the main outcome variables identified for this study analysis are per capita income, health expenditure, food expenditure, household family size, reported illness in the past two months, days of absence from work or daily activity due to illness in the past two months, number of reported death in the last 12 months, presence of orphan, and number of orphans in the household.

The probit regression model was used to estimate the propensity scores:

\section{Health status $=(\beta 0+\beta 1$ educ $+\beta 2$ gender dummy $+\beta 3$ age + \\ $+\beta 4$ occupation dummy $1+\beta 5$ occupation dummy $2+$ $+\beta 6$ occupation dummy $3+\varepsilon)$}

where $\beta 0$ is constant and $\beta 0, \beta 1, \beta 2, \beta 3, \beta 4, \beta 5 \wedge \beta 6$, are coefficients, and the health status is the dependent variable, which takes the number 1 if HIV-affected, 0 otherwise.
Table 1. The percentage of treated (HIV-affected) and controlled (non-HIV-affected) household samples

\begin{tabular}{l|c|c}
\hline Health status & Frequency & Percent \\
\hline Non-HIV & 91 & 37.92 \\
\hline HIV-affected & 149 & 62.08 \\
\hline Total & 240 & 100 \\
\hline
\end{tabular}

The treatment condition of being HIV/AIDS or not is represented by 1 if HIV patient households and 0 if nonHIV households. The intention here is to match 149 HIV patients group with 91 non-HIV households and compare their outcomes. The sample size and percentage of these two groups are indicated in Table 1.

The study used four common matching methods widely used in the evaluation literature. The methods employed were the nearest-neighbor, radius or caliper, Kernel and stratification method $[6,14,16]$. These methods all yielded very similar estimates of the impact of HIV/AIDS on the outcome variables. They help to assess 'nearness' between control and treated cases [6] and help to estimate the average treatment effect on the treated (ATT) [15]. ATT is the difference between the outcomes of treated and the outcomes of the treated observations, if they had not been treated [16]. Given a population of units denoted by $i i$, if the propensity score $p\left(X_{i}\right) p\left(X_{i}\right)$ in the above equation is known, then the average effect of treatment on the treated (ATT) can be estimated as follows [16]:

$$
\begin{gathered}
T \equiv \mathrm{E}\left\{Y_{1 i}-Y_{0 i} \mid D_{i}=1\right\} \\
=E\left[E\left\{Y_{1 i}-Y_{0 i} \mid D_{i}=1, p\left(X_{i}\right)\right\}\right] \\
=E\left[E\left\{Y_{1 i} \mid D_{i}=1, p\left(X_{i}\right)\right\}-E\left\{Y_{0 i} \mid D_{i}=0, p\left(X_{i}\right)\right\} \mid D_{i}=1\right]
\end{gathered}
$$

where the outer expectation is over the distribution of $\left(p\left(X_{i}\right) D_{i}=1 p\left(X_{i}\right) D_{i}=1\right), Y_{1 i} Y_{1 i}$ and $Y_{0 i} Y_{0 i}$ are the potential outcomes in the two counterfactual situations of (respectively) treatment and no treatment. In this study, ATT will represent average HIV/AIDS effect on the HIV patient households.

\section{Results}

Results are relatively consistent across the four algorithms. The most efficient algorithms (i.e. capturing the highest number of significant outcomes) is the stratification matching but the estimated outcomes are similar in magnitude.

The study results suggested that the monthly total health expenditure of HIV-affected households is higher than that of the non-affected counterparts. However, the result is statistically significant only when using the stratification method as shown in Table 2. In this estimate, the total monthly health expenditure of the affected households is higher than the non-affected on average by 375 birrs (about $\$ 18$, 
Table 2. Main findings on the effects of HIV/AIDS (average treatment effect) on the households in Addis Ababa

\begin{tabular}{|c|c|c|c|c|}
\hline Outcome variables & Nearest-neighbor & Kernel & Stratification & Radius \\
\hline Family size of the household & $-0.361(0.582)$ & $-0.424(0.510)$ & $-0.404(0.507)$ & $-0.375(0.589)$ \\
\hline $\begin{array}{l}\text { Reported illness of two months } \\
\text { (yes, response in \%) }\end{array}$ & $0.266^{*}(0.159)$ & $0.208(0.134)$ & $0.281^{* *}(0.095)$ & $0.259(0.160)$ \\
\hline Lost work days (No of days) & $6.448^{*}(3.853)$ & $6.365^{\star \star}(2.655)$ & $5.289^{* *}(0.598)$ & $6.375(3.881)$ \\
\hline Reported death (yes response in \%) & $0.162(0.130)$ & $0.087(0.097)$ & $0.116^{*}(0.020)$ & $0.152(0.131)$ \\
\hline Presence of orphan (yes response in \%) & $-0.019(0.151)$ & $-0.019(0.109)$ & $-0.068(0.105)$ & $-0.019(0.154)$ \\
\hline Monthly per capita income & $-294.308(466.385)$ & $-388.614(471.701)$ & $-460.794^{* *}(192.107)$ & $-298.419(469.816)$ \\
\hline Monthly total health expenditure & 318.419 (313.676) & $130.032(394.626)$ & $374.834^{\star *}(315.158)$ & $299.557(315.823)$ \\
\hline Total monthly household expenditure & $-881.123(635.130)$ & $-1165.74^{*}(605.364)$ & $-1087.753^{* *}(727.322)$ & $-918.25(639.544)$ \\
\hline $\begin{array}{l}\text { Per capita total monthly } \\
\text { expenditure }\end{array}$ & $-46.789(546.071)$ & $-98.758(399.530)$ & $-210.414(170.034)$ & $-51.598(550.051)$ \\
\hline Per capita monthly food expenditure & $-136.52(193.876)$ & $-121.292(201.194)$ & $-165.043^{\star \star}(32.998)$ & $-138.219(195.297)$ \\
\hline Share of expenditure on food & $-18.98^{\star * *}(7.026)$ & $-13.163^{*}(7.27)$ & $-14.919^{\star * \star}(4.122)$ & $-18.839^{\star * *}(7.076)$ \\
\hline $\begin{array}{l}\text { Share of health expenditure } \\
\text { on total expenditure }\end{array}$ & $14.69^{\star \star \star}(5.21)$ & $11.789^{*}(6.68)$ & $12.430^{* * *}(3.699)$ & $14.320^{\star \star \star}(5.253)$ \\
\hline $\begin{array}{l}\text { Per capita monthly health } \\
\text { expenditure }\end{array}$ & $136.45(113.022)$ & $101.061(135.251)$ & $118.379^{* *}(43.347)$ & $134.115(113.905)$ \\
\hline $\begin{array}{l}\text { Share of non-food (utilities) } \\
\text { expenditure }\end{array}$ & $4.287(5.76)$ & $1.373(6.278)$ & $2.489(5.681)$ & $4.519(5.802)$ \\
\hline
\end{tabular}

the average per capita income is around $\$ 75$ in the sample). The share of health expenditure on the total expenditure is also higher for the treated households. The result shows that the share of health expenditure on total expenditure is statistically significant in all matching methods used in the study. The study finds that the HIV-affected households have approximately a $14 \%$ higher share of health expenditure on total expenditure. These results confirm that HIV/AIDS generates significantly higher health expenses for the affected households. The results hold also when considering household sizes, and the per capita monthly health expenditure is higher for the affected households and it is statistically significant as shown in Table 2.

The estimate of the treatment effect on the monthly per capita income indicates that after matching the HIV-affected households still have lower levels of monthly per capita income than the non-affected households. The affected households on average have 460 birrs lower monthly per capita income, as compared to the non-affected households. This figure was significant at 5\% significance level when applying stratification matching. In addition, the result suggested that after matching, the affected households have lower total monthly expenditure. Accordingly, the average treatment effect on the treated was approximately 1,120 birrs (around U.S. \$54). This means the total household expenditure of the affected household is lower by 1,120 birrs. The result also suggested that per capita total monthly expenditure is lower among the treated case. However, this result is found to be non-significant in all matching methods as shown in Table 2, which presents the main findings on the effects of HIV/AIDS on the households in Addis Ababa, Ethiopia, considering a variety of outcome variables after matching with different algorithm.

The per capita monthly food expenditure is also lower in the treated group. The HIV-affected households' per capita monthly food expenditure is lower on average by 165 birrs. The average HIV effect on the affected household is most prominent for the share of expenditure on food. As shown in the Table, the share of food expenditure among the affected households found to be lower than the non-affected. The average treatment effect on the treated is lower by 13 to $19 \%$. This means the HIV-affected households have a lower share of expenditure on food. This result is statistically significant in all matching methods used in this study. The result suggested that HIV/AIDS has a strong impact on the food consumption level of the affected households. This is a result, which is consistent with the fact that health expenditure erodes food expenditure, especially in poor households with tighter budget constraints. Instead, the share of non-food or utility expenditure is found to be statistically insignificant in all matching methods.

The average workdays lost because of illness in the HIVaffected households were relatively higher than the nonaffected households. The HIV-affected households had lost, on average, 6 more workdays due to illness within two months period. The result of average treatment effects is significant in most matching methods. This result suggested that 
either the affected households spend more hours at home because of HIV or they might have spent their time in caregiving activities for the household members.

After the result of nearest neighbor and stratification, matching (see Table 2), HIV-affected households report more illness within the period of two months before the survey of this study. The households were approximately $28 \%$ more likely to report illness in two months. The difference in reported death of the two groups of households is not statistically significant in all matching methods. This report suggested that affected households were approximately $11 \%$ more likely to report a death within one-year period before the survey of this study.

The result also suggested that there are no statistically significant differences on the household responses to the presence of orphan in their households. Before propensity score matching, $34 \%$ of the HIV-affected household respondents reported that they have an orphan in their household. The figure was $13 \%$ for the non-affected households, and the difference was significant. However, after propensity score matching, the result indicates that the HIV-affected households were not likely to have more orphan the nonaffected households, as shown in the findings of this study. This result may reflect the fact that our definition of HIVaffected households refers to members of the household currently affected by HIV/AIDS and, as also reflected by the non-significant difference in reported death, it would seem that the higher presence of orphans in the target group before matching is more likely to depend from other characteristics of this household rather than HIV/AIDS.

Household family size was one of the outcome variable considered in this study. However, the result suggests that HIV/AIDS is not affecting the family size of the households. As shown in Table 2 in all matching methods, the difference in the mean household size between HIV-affected and non-affected households were not significant. Before matching HIV-affected, households have on average 3.6 family size and for non-affected households, it was 3.03. This figure was not significant as well. This is again consistent with the above consideration about deaths and orphan size.

\section{Discussions}

The results of this study on the economic burden of HIV/AIDS at the household level in Addis Ababa indicate that the affected households have lower monthly per capita income, a higher share of health expenditure, and a lower share of food expenditure on total expenditures. The result revealed that the households have on average have 460-birr lower monthly per capita income (about 25\% less), $14 \%$ higher share of health expenditure, and $19 \%$ lower share of expenditure on food. All of these will have possible consequences on the household welfare in particular, and on the health system in general. The findings of this study are consistent with other studies in sub-Saharan African countries. For example, a study by Alam and Mahal [17] showed that in South Africa, the income is lower from 35\% to $50 \%$ in HIV-affected households [8]. Russell [3] found that the mean direct cost of HIV/AIDS was more than $10 \%$ of the household income. This estimate was considered 'catastrophic' to the household. The same study confirmed that the direct costs related to HIV/AIDS like treatment and funeral costs were likely to be catastrophic. One study in Nigeria also estimated that direct private healthcare costs and indirect income loss were approximately $56 \%$ of annual income per capita in the affected households [6].

In sub-Saharan African countries, the HIV-affected individuals and their families lose a significant portion of their workdays due to HIV/AIDS and related illnesses. The result from this paper suggested that HIV-affected households in Addis Ababa lost more workdays due to HIV. The estimated average work days lost due to illness in this study is 6 days within two months period. This result is comparable with the previous studies in SSA. For example, in Tanzania, HIV-positive women lost higher work days of 429, and HIV-positive males lost 297 days within 18 months period [3]. A study in Nigeria [6] found that approximately 16 additional workdays were lost among HIV-positive individuals by using the matched sample in their analysis.

To cope with this economic burden of HIV, the households may use different coping strategies. The result of this paper reveals that sale of items is the major coping mechanism, while the affected households need HIV and related healthcare expenditures.

The sample data from this study indicates that more than $30 \%$ of the affected households sold their assets. However, for the non-affected households, this figure was considerably lower, which accounts for approximately $7 \%$. A similar study in Nigeria estimated that $13 \%$ of the HIV-positive and $2.5 \%$ HIV-negative individuals and their household members sold their items to pay for illness-related expenses [6]. Such a strategy will affect households' welfare by depleting their resources, reducing their income and food consumption levels $[2,3,5]$.

\section{Conclusions}

The paper analyzed the economic burden of HIV/AIDS on the households in Addis Ababa by comparing HIV-affected households with non-affected. Since the characteristics of these two groups are likely to differ because of other reason than HIV/AIDS, a propensity score matching approach to control for selection biases was used. Based on the result of the study, the following conclusions are drawn:

HIV/AIDS has a high impact on households' health care expenditure and income. The HIV-affected households have considerably lower monthly income (about 25\%) and higher health expenditure (about twice as higher). HIV affects the households' income by increasing absence days from work and caregiving activity to the affected person in the households.

Sale of the household assets was the main strategy of the affected households to cope with the financial costs of healthcare. In addition, the HIV-affected households do not have adequate and sustainable mechanisms to cope with the financial costs of illness. The households struggle to cope 
with strategies that damage their asset portfolio, and this can lead to impoverishment. HIV/AIDS affected the food consumption level of the household. The affected households have a lower share of food expenditure. HIV/AIDS is not affecting the family size and the presence of orphans between the households in this study.

Overall, the economic burden of HIV/AIDS falls significantly on affected households compared to non-affected households. Based on the survey result and conclusions, this study supports the following recommendations aimed at reducing the economic burden of HIV/AIDS on households in Addis Ababa. Both governmental health policymakers and non-governmental donor organizations intervention needs to mitigate the economic burden of HIV/AIDS, especially on the affected and poor households in Addis Ababa.

Firstly, an alternative health care financing mechanism should have been designed and introduced to the households to reduce the health expenditure borne by them. Currently, most of the households pay for the healthcare service by out of pocket expenses at the time of service needed. This can affect the households' income and health when they are severely ill or when they are unable to pay. The HIV-affected households may pay more for the healthcare services. However, if there is a form of prepayment mechanism, whether it is formal or informal insurance scheme, the health expenditure at the time of healthcare use will be reduced and the households' income will not be affected. This means there would be a mechanism of risk sharing from severely ill to healthy and cost sharing from rich to poor. In this case, the households will not pay too much when they are severely ill. Yet again, this promotes equity in access to healthcare.

Secondly, the government should strengthen the households' economic activity by creating job opportunities for the affected individuals to support them and their families. Developing different income generating sources through microcredit enterprises and promoting access to credit facilities for affected households would open the chance to take loans to finance their livelihood and to cover for their healthcare expenses at the time of service needed. The micro saving and credit associations should also give priority to the affected households to provide loan facilities to them.

Thirdly, donors and international non-governmental organizations who work in the HIV/AIDS program should support the HIV-affected households by providing food to them. Since most of the affected households allocate majority of their income to buy the healthcare service, their food consumption level is severely affected. However, if the donor organizations would help them by providing food, especially to the most vulnerable household members, their food consumption level would improve.

Finally, further quantitative microeconomic research on the burden of HIV/AIDS and other epidemic is needed, especially by focusing the most vulnerable households to improve their living standard and health conditions.

\section{Conflict of interest}

The authors declare no potential conflicts of interest with respect to the research, authorship, and/or publication of this article.

\section{References}

1. Aye R, Wyss K, Abdualimova H, Saidaliev S. Household costs of illness during different phases of tuberculosis treatment in Central Asia: a patient survey in Tajikistan. BMC Public Health 2010; 10: 18.

2. McIntyre D, Thiede M, Dahlgren G, Whitehead M. What are the economic consequences for households of illness and of paying for health care in low- and middle-income country contexts? Soc Sci Med 2006; 62: 858-865.

3. Russell S. The economic burden of illness for households in developing countries: a review of studies focusing on malaria, tuberculosis, and human immunodeficiency virus/acquired immunodeficiency syndrome. Am J Trop Med Hyg 2004; 71 (2 Suppl): 147-155.

4. Xu K. Catastrophic health expenditure. Lancet 2003; 362: 997.

5. Sauerborn R, Adams A, Hien M. Household strategies to cope with the economic costs of illness. Soc Sci Med 1996; 43: 291-301.

6. Mahal A, Canning D, Odumosu K, Okonkwo P. Assessing the economic impact of HIV/AIDS on Nigerian households: a propensity score matching approach. AIDS 2008; 22 (Suppl 1): S95-S101.

7. UNICEF. 'The State of the World's Children 2005 - Childhood under Threat. UNICEF Publications, UNICEF'. N.p., 2015 (Accessed: 2 June 2015).

8. HIV and AIDS: AiDS Orphan. 2013. Available from: https://www. aidsorphan.net/

9. FHAPCO (Federal Ministry of Health / National HIV/AIDS prevention and control office). AIDS in Ethiopia. Addis Ababa, 2005.

10. City Administration Health Bureau. HIV/AIDS in Addis Ababa. Background, Projections, Impacts, and Interventions. Addis Ababa, 1999.

11. Central Statistical Agency. Ethiopia Demographic and Health Survey. Preliminary Report. Addis Ababa, 2011.

12. Bollinger L, Stover J, Seyoum E. The Economic Impact of AIDS in Ethiopia. The Futures Group International, 1999.

13. Rosenbaum P, Rubin D. The Central Role of the Propensity Score in Observational Studies for Causal Effects. Biometrika 1983; 70: 41.

14. Bai H. A comparison of propensity score matching methods for reducing selection bias. Int J Res Meth Educ 2011; 34: 81-107.

15. Gnawali D, Pokhrel S, SiÃ A, et al. The effect of community-based health insurance on the utilization of modern health care services: Evidence from Burkina Faso. Health Policy 2009; 90: 214-222.

16. Becker OS, Ichino A. Estimation of average treatment effects based on propensity scores. The Stata Journal 2002; 2: 358-377.

17. Alam K, Mahal A. Economic impacts of health shocks on households in low and middle-income countries: a review of the literature. Global Health 2014; 10: 21. 